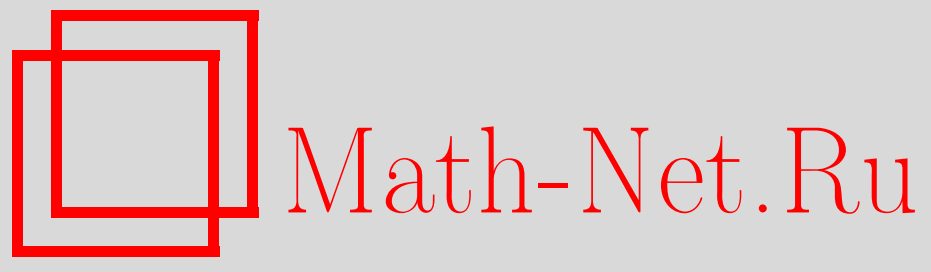

М. И. Штогрин, Изометрические вложения поверхностей платоновых тел, УМН, 2007, том 62, выпуск 2, 183184

DOI: https://doi.org/10.4213/rm6386

Использование Общероссийского математического портала Math-Net.Ru подразумевает, что вы прочитали и согласны с пользовательским соглашением http: //www . mathnet.ru/rus/agreement

Параметры загрузки:

IP : 54.172 .240 .79

26 апреля 2023 г., 13:07:50

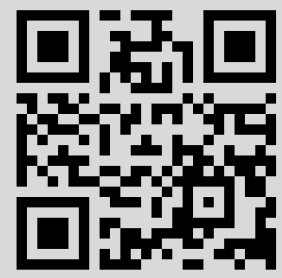




\section{Изометрические вложения поверхностей платоновых тел}

\section{М. И. Штогрин}

Применяя метод А.В. Погорелова [1], в [2] даны некоторые кусочно гладкие погружения (вложения) поверхностей платоновых mел в $\mathbb{R}^{3}$. А именно, любой пояс, составленный из замкнутых плоских геодезических поверхности, расположенных в попарно параллельных плоскостях, допускает кусочно гладкие изгибания, описанные в [1]; пояс представляет собой боковую поверхность призмы, антипризмы или трапец,эдра. Но некоторые грани октаэдра, куба, икосаэдра, додекаэдра остались плоскими [2]. Ниже предъявлены кусочно гладкие вложения поверхностей платоновых тел в $\mathbb{R}^{3}$ без плоских кусков.

Для реального применения метода Погорелова потребовалась предварительная подготовка, так как специальные изометрические преобразования цилиндрической поверхности Погорелов фактически не задавал - он строил готовый образ при изометрическом преобразовании эвристически, см. $[1 ; \S 3$, п. 1]; для его целей было вполне достаточно этого. Для наших целей нужно знать не только образ, но и удобный способ получения его из прообраза. Поэтому метод Погорелова был всесторонне проанализирован: проведено исследование прообразов криволинейных ребер при преобразовании, открытом Погореловым. Прообразы криволинейных ребер рассматривались не на самой поверхности цилиндра, а на ее развертке $R \in \mathbb{R}^{2}$.

Сказанное является очень важным в изложении метода Погорелова с целью его практического применения. И, самое главное, анализ открытых Погореловым изометрических преобразований цилиндра привел к следующему неожиданному результату: существуют специальные изометрические преобразования конуса, аналогичные преобразованиям цилиндра. Это позволило доказать следующую теорему.

Теорема. Поверхности платоновых тел допускают кусочно гладкие изометрические погружения в трехмерное евклидово пространство $\mathbb{R}^{3}$ без плоских кусков.

До тех пор, пока не были обнаружены специальные изометрические преобразования конуса, аналогичные специальным изометрическим преобразованиям цилиндра, открытым и исследованным Погореловым, см. [1; 33, п. 1], автор работы [2] тщетно пытался доказать гипотезу о том, что поверхность платонова тела допускает кусочно гладкие изгибания не более чем одного пояса. Тогда автору казалось, что поверхность платонова тела не может быть искривлена полностью, оставшись без малейших плоских кусков, если тело не является тетраэдром.

Обнаружение специальных изометрических преобразований конуса, обобщающих специальные изометрические преобразования цилиндра, является существенным продвижением с точки зрения обобщения метода Погорелова [1]. По каким-то странным обстоятельствам специальные изометрические преобразования конуса не были обнаружены Погореловым. Причина этого неизвестна и остается неразгаданной тайной ведь он был в двух шагах от четко поставленной цели.

Доказательство теоремы в случае тетраэдра дано в работе [2]. Мы остановимся на этом подробнее. Нас интересует не только сам факт существования погружения, но и способ его построения. Для этой цели займемся разверткой тетраэдра.

Рассмотрим произвольный прямоугольник. Отождествим точки любой его стороны, симметричные относительно середины. Получим развертку поверхности трехмерного симплекса с группой симметрии $\overline{4} 2 m$, см. рис. 1 , а). Порядок группы втрое больше при отношении сторон прямоугольника $\sqrt{3}: 1$, когда симплекс правильный.

Возьмем четверть развертки, рис. 1, b). Отрежем два равнобедренных прямоугольных треугольника. Останется параллелограмм, рис. 1, с). Заменим диагональ

Работа выполнена при финансовой поддержке Российского фонда фундаментальных исследований (грант 05-01-00170, 06-01-72551-НЦНИЛ, 06-01-90845-Мол), программы "Ведущие научные школы” (грант НШ-4182.2006.1) и государственной программы фундаментальных исследований ОМН РАН “Современные проблемы теоретической математики”. 
на гладкую кривую, рис. 1, d): она лежит внутри параллелограмма; направление касательной в любой точке кривой расположено внутри острого угла, рис. 1, с).

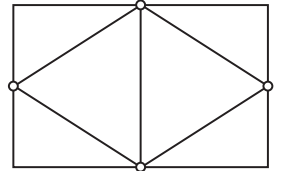

a)

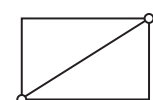

b)

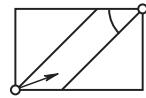

c)

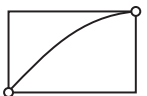

d)

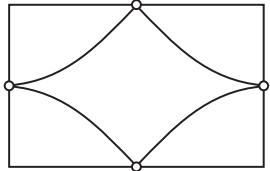

e)

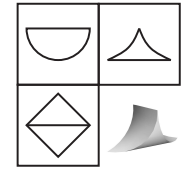

f)

Рис. 1. Симплекс. Развертки а), е); четверти b)-d); кривое вложение f)

Размножив кривую зеркалами, рис. 1, е), получим развертку кусочно гладкой поверхности, изометричной поверхности симплекса. Для модели удобно отношение сторон прямоугольника $\pi: 2$; тогда в качестве кривой возьмем выпуклый участок стандартной синусоиды (от перегиба до максимума), рис. 1, d); все три гладких куска поверхности суть части круговых цилиндров, рис. 1, f).

ЗАмЕчАниЕ. Если угловой коэффициент касательной к гладкой кривой в ее концах равен 1, то кусочно гладкая поверхность имеет лишь две криволинейные грани.

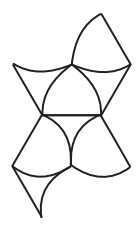

a)

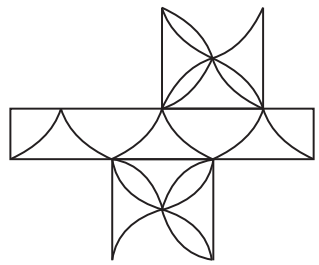

b)

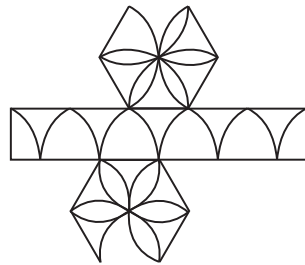

c)

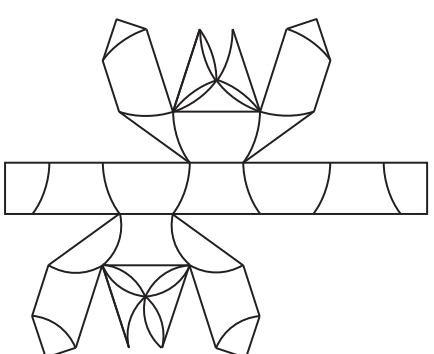

d)

Рис. 2. Развертки: октаэдра а), куба b), икосаэдра с), додекаэдра d)

Возьмем диаметрально противоположные вершины $S$ и $N$ октаэдра, куба, икосаэдра, додекаэдра. Перпендикулярно отрезку $S N$ проведем плоские сечения, проходящие через вершины. Ободки поверхности, расположенные между соседними плоскостями, суть боковые поверхности пирамиды, антипризмы, трапецоэдра, усеченной пирамиды с усеченными ребрами. Ободок имеет группу изометрий с $n$ плоскостями зеркальной симметрии, проходящими через общую ось, $n=4,3,5,3$ соответственно. Заменим прообраз одного бокового ребра ободка на дугу (прообраз гладкого ребра) и размножим ее отражениями от следов зеркал на развертке ободка. Дугу можно взять достаточно близкую к прообразу ребра, скажем, составляющую с ним выпуклый сегмент. Тогда на развертке ободка будут чередоваться выпуклые и невыпуклые участки, см. рис. $2, \mathrm{a})-\mathrm{d})$. При кусочно гладком вложении в $\mathbb{R}^{3}$ любой гладкий кусок будет направлен выпуклостью внутрь (наружу) поверхности, если его прообраз на развертке является (не)выпуклым.

\section{Список литературы}

[1] А. В. Погорелов, Изгибания поверхностей и устойчивость оболочек, Наукова думка, Киев, 1998. [2] М. И. Штогрин, УМН, 60:4 (2005), 221-222.

М. И. Штогрин (M. I. Shtogrin)

Математический институт им. В. А. Стеклова РАН

E-mail: stogrin@mi.ras.ru
Представлено В. М. Бухштабером Принято редколлегией 27.12 .2006 